\title{
AUFSÄTZE
}

\section{Erfolgswertgleichheit als fixe Idee oder: Zurück zu Weimar? Zum Urteil des Bundesverfassungsgerichts über das Bundeswahl- gesetz vom 3. Juli 2008}

\author{
Dieter Noblen
}

Im Jahre 2008 hat sich das Bundesverfassungsgericht (BVerfG) erneut mit dem Wahlrecht zum Deutschen Bundestag befassen müssen. Dieses Mal lagen zwei Wahlprüfungsbeschwerden von Wilko Zicht und Martin Fehndrich vor, die in den widersinnigen Wirkungen der Überhangmandate im System der personalisierten Verhältniswahl die Grundsätze der Gleichheit und Unmittelbarkeit der Wahl verletzt sahen. ${ }^{1}$

Bekanntlich treten Überhangmandate im bundesdeutschen Wahlsystem dann auf, wenn eine Partei mit den Erststimmen in den Wahlkreisen mehr Mandate erringt, als ihr nach den Zweitstimmen entsprechend den auf die Landesliste entfallenden Mandaten zustehen. Den Überhangmandaten wurde, nachdem einerseits das BVerfG sie 1957 als systembedingt und mit dem Gleichheitsgrundsatz vereinbar erklärt hatte ${ }^{2}$, und sie andererseits in den Wahlen von 1965 bis 1987 nur gelegentlich und in geringer Zahl auftraten, kaum noch Aufmerksamkeit zuteil. Das änderte sich schlagartig, als 199416 Überhangmandate zustande kamen und zwölf davon auf die CDU entfielen. Erst die Überhangmandate bescherten der siegreichen Koalition von CDU/CSU und FDP eine satte parlamentarische Mehrheit. Obwohl unter dem Gesichtspunkt der Sicherung parlamentarischer Regierung dieser Effekt der personalisierten Verhältniswahl positiv hätte konnotiert werden können, traten nun erneut Zweifel an der Übereinstimmung der Überhangmandate mit dem Gleichheitsgrundsatz im Wahlrecht auf. Das BVerfG entschied in seinem Urteil vom 10. April 1997, dass der Gesetzgeber einen Mandatsausgleich vorsehen kann, dieser aber für die Verhältniswahl personalisierten Typs nicht zwingend sei. ${ }^{3}$ Das Gericht erkannte an, dass der Gesetzgeber mit der Einrichtung der Fünfprozentklausel Begrenzungen der Proportionalität gewollt hat. Das Proporzprinzip sei demnach keine absolute Richtschnur für die Bewertung einzelner Elemente der personalisierten Verhältniswahl, also auch nicht der Überhangmandate. Das Gericht hielt seinerzeit die überkommene Regelung für rechtens, wenn dafür Sorge getragen würde, dass sich die Zahl der Überhangmandate in Grenzen hielte.

1 BVerfG 2 BvC 1/07 und 2 BvC 7/07, Beschluss vom 3. Juli 2008 (Negatives Stimmgewicht), im Weiteren zitiert in der Fassung der Internet-Veröffentlichung unter Hinweis auf die dortigen Randnummern; vgl. auch Abdruck in: NJW 2008, S. 2700; DVBl 2008, S. 1045; NVwZ 2008, S. 991.

2 BVerfG 2 BvR 9/56, Beschluss vom 3. Juli 1957 (Listenwahl); BVerfGE 7, S. 63; vgl. DÖV 1957, S. 715.

3 BVerfG 2 BvC 3/96, Beschluss vom 10. April 1997 (Grundmandatsklausel); BVerfGE 95, S. 408 ff.; vgl. auch Abdruck in: NJW 1997, S. 1568; DVBl 1997, S. 784; DÖV 1997, S. 595; BayVBl 1997, S. 429. 
Gut zehn Jahre später sah sich das BVerfG nun jedoch in derselben Angelegenheit mit einer veränderten Argumentation konfrontiert. Zwar war bereits vordem bekannt, dass Überhangmandate dazu führen können, dass eine Partei bei einem höheren Zweitstimmenanteil eines Mandates verlustig gehen kann ${ }^{4}$ - der Fall wurde aber erst bei einer Nachwahl im Wahlkreis Dresden 2005 akut. Erhärtet und allgemein publik wurde, dass es für eine Partei mit Überhangmandaten von Vorteil sein kann, weniger Stimmen in einem Bundesland zu bekommen, wenn dadurch die Mandatszahl in der bundesweiten Verteilung der Mandate an die Parteien (Oberverteilung) nicht negativ betroffen ist. Einfluss kann die niedrigere Stimmenzahl auf die Verteilung der Mandate auf die einzelnen Landeslisten der Parteien (Unterverteilung) nehmen, denn eine niedrigere Anzahl an Zweitstimmen kann hier bewirken, dass eine andere Landesliste mit einem Mandat profitiert. Verliert die Partei in einem Bundesland an Zweitstimmen, in dem sie ein Überhangmandat erzielt hat, erleidet sie keinen Nachteil, weil ihre Liste ohnehin keinen Anspruch auf ein Mandat gehabt hätte, sie die von ihr erzielten Direktmandate aber nicht verlieren kann. Eine andere Landesliste kann aber ein Mandat hinzugewinnen. Damit erhöht auch die betreffende Partei ihren Mandatsanteil auf Bundesebene. Der umgekehrte Effekt ist ebenfalls denkbar. Eine Partei kann durch ein Mehr an Zweitstimmen in einem Bundesland ein Überhangmandat verlieren und damit auf der Bundesebene ebenfalls ein Mandat einbüßen.

Dieses „negative Stimmgewicht“ beziehungsweise diesen ,inversen Erfolgswert" erklärte das höchste Gericht am 3. Juli 2008 für verfassungswidrig. Ein Verfahren, das (entsprechend $\$ 7$ Abs. 3 und $\$ 6$ Abs. 4 und 5 des Bundeswahlgesetzes) ermöglicht, „dass ein $\mathrm{Zu}$ wachs an Zweitstimmen zu einem Verlust an Sitzen der Landeslisten oder ein Verlust an Zweitstimmen zu einem Zuwachs an Sitzen der Landeslisten führen kann "5, verletzt nach Ansicht des Gerichts Art. 38 Abs. 1 Satz 1 GG. Der Gesetzgeber wurde verpflichtet, „spätestens bis zum 30. Juni 2011 eine verfassungsgemäße Regelung zu treffen"6.

\section{Bewertung des Urteils}

Im Ergebnis ist dem Bundesverfassungsgericht durchaus zuzustimmen. Der Vertreter der Verteidigung hatte zwar in der Linie der ständigen Rechtsprechung des Gerichts argumentiert, dass Abweichungen vom Proporz im System der personalisierten Verhältniswahl zulässig seien und dabei auf die Fünfprozentklausel verwiesen, die das reine Proporzprinzip durchbricht. Auch hatte er betont, dass für den Wähler zwar erkennbar sein müsse, „wie sich seine Stimme auf Erfolg oder Misserfolg der Wahlbewerber auswirken könne. Dies könne aber nur im Sinne einer Kenntnis der theoretisch denkbaren Wirkungen zu verstehen sein, da die tatsächlichen Wirkungen stets nur im Zusammenhang der Stimmabgabe aller Wähler eintreten könnten“. Er hatte dabei jedoch nicht bemerkt, dass es sich beim inversen Erfolgswert um etwas anderes handelt, was das Gericht klar erkannte: Die Wirkungen einer Sperrklausel sind vorhersehbar. Wählerstimmen für Parteien, die die Schwelle

4 Vgl. Hans Meyer, Der Überhang und anderes Unterhaltsames aus Anlass der Bundestagswahl 1994, in: KritV, 77. Jg. (1994), H. 4, S. 312 - 362; Joachim Behnke, Ein integrales Modell der Ursachen von Überhangmandaten, in: PVS, 44. Jg. (2003), H. 1, S. $41-65$.

5 BVerfG 2 BvC 1/07 und 2 BvC 7/07, a.a.O. (Fn. 1), Rn. 140.

6 Ebenda, Urteilsspruch. 
überschreiten, haben den gleichen Erfolgswert. Wie sich die Zweitstimmen auswirken, welche den inversen Erfolgswert auslösen, ist hingegen für den Wähler nicht vorhersehbar.

Der inverse Erfolgswert ist ein Effekt des im Wahlsystem zum Bundestag vorliegenden spezifischen Verfahrens, nach dem die Anrechnung der Direktmandate auf die proportional an die Parteien verteilten Mandate erfolgt. Diese Regelung ist vor allem bedenklich, weil sie willkürliche Ergebnisse hervorrufen kann. Der Wähler ist im Grunde nicht mehr Herr seiner Stimme. Er verbindet mit seiner Stimmabgabe eine bestimmte Intention, die im Verfahren der Anrechnung der Direktmandate jedoch ins Gegenteil verkehrt werden kann. Dem System mangelt es folglich an Transparenz. Der Wähler kann seine Stimme nicht gezielt einsetzen. Er verliert das Vertrauen in das Wahlsystem, das folglich an Legitimität einbüßt. Transparenz und Legitimität sind aber entscheidende Maßstäbe zur Messung der Qualität eines Wahlsystems. Deshalb ist eine Reform der personalisierten Verhältniswahl, die die Anrechnung der Direktmandate auf die den Parteien proportional zugewiesenen Mandate neu regelt, dringend erforderlich.

Gewiss, Wahlsysteme leben mit Paradoxien ihrer Ergebnisse (dazu weiter unten mehr). Die Forderung nach einer Reform wird im vorliegenden Falle dadurch erleichtert, dass das negative Stimmgewicht keine unabdingbare Folge der mit der Personenwahl verbundenen Verhältniswahl ist, da Überhangmandate in diesem Wahlsystemtyp nicht zwangsläufig auftreten müssen. Man kann ihr Entstehen von Vorneherein ausschließen, man kann sie aber auch proportional gegenrechnen, so dass sie weder einen die proportionale Vertretung der Parteien beeinträchtigenden Effekt haben, noch den Wähler in undurchsichtige Wahlhandlungen verwickeln können. Es geht um eine Korrektur eines Elements, eines Verfahrens innerhalb der personalisierten Verhältniswahl, nicht um eine weitreichendere Reform. Ob diese durch den Karlsruher Richterspruch nun obligate Korrektur allerdings zum Anlass genommen werden wird, über eine grundsätzlichere Wahlsystemreform nachzudenken, steht auf einem anderen Blatt. Selbst das BVerfG deutet mit dem Hinweis, dass eine Reform im Sinne eines Grabensystems verfassungskonform sei, Reformüberlegungen jenseits des Typs der personalisierten Verhältniswahl an.

\section{Zur Urteilsbegründung allgemein}

Kann man im Ergebnis das Urteil des Gerichts bejahen, so ergeben sich, wie gerade dargelegt, nicht nur unterschiedliche Begründungen, sondern einige Argumente des Bundesverfassungsgerichts sind wahlsystemtheoretisch höchst bedenklich. Zunächst fällt auf, dass begrifflich keine Unterscheidung zwischen Wahlrecht und Wahlsystem getroffen wird. Das leistet der im Urteil vorherrschenden Tendenz Vorschub, Anforderungen an das individuelle Wahlrecht und die Wahl als Kreationsvorgang einer repräsentativen Versammlung mit denen an ein Wahlsystem gleichzusetzen.

Das Wahlrecht, also das Recht zu wählen und gewählt zu werden, kennt klare Prinzipien, deren Verletzung relativ einfach zu diagnostizieren und zu bewerten ist. Beim Merkmal „allgemein“ gilt es zu prüfen, ob tatsächlich alle Bürger das Wahlrecht genießen, also in die Wählerlisten eingetragen sind; beim Merkmal „gleich“, ob jeder Wähler dasselbe Stimmgewicht, also keiner mehr Stimmen als ein anderer hat. Mehrstimmenrecht ist ausgeschlossen. Beim Merkmal „direkt“ ist zu fragen, ob der Wähler unmittelbar einen Kandidaten oder eine Partei wählt, nicht erst eine Person oder ein Gremium, das dann die eigentli- 
che Wahl des Mandatsträgers vornimmt. Diese Verfassungsprinzipien eines demokratischen Wahlrechts sind nicht ohne Weiteres auf das Wahlsystem übertragbar. Zumal die dogmatische Handhabung von Wahlrechtsprinzipien eignet sich weniger zur Bewertung von Wahlsystemfragen. Es sind andere Maßstäbe, die zur Geltung kommen, beispielsweise die Erfüllung von Funktionen, die von Wahlsystemen erwartet wird, die Legitimität, die Wahlsysteme beanspruchen können und tatsächlich genießen. Des Weiteren gibt es in Wahlsystemen keine optimale Lösung. Sie haben Vorteile und Nachteile. Es gibt darüber hinaus unendlich viele Lösungsmöglichkeiten, wie Wähler ihre politischen Präferenzen in Wählerstimmen ausdrücken und diese in Mandate übertragen werden können. Es gilt also abzuwägen und politisch zu entscheiden, wobei der jeweilige Kontext eine große Rolle spielt. All dies ist beim Wahlrecht, dem Recht zu wählen und gewählt zu werden, nicht der Fall.

Es ist verständlich, dass ein Gericht sich fester Prinzipien bedient, um Recht zu sprechen. Da sich das Grundgesetz zum Wahlsystem nicht äußert, ist auch die Tendenz des Bundesverfassungsgerichts verständlich, die klassischen Prinzipien des Wahlrechts auf die Wahlsystemmaterie zu übertragen. So wird argumentiert, dass für das Wahlsystem die Gleichheit und die Unmittelbarkeit der Wahl wesentliche Kriterien der Beurteilung des Wahlsystems beziehungsweise seiner Verfassungskonformität seien. Deren Anwendung in einzelnen Fällen hat auch im juristischen Lager stets zu Kritik an der Argumentation des Bundesverfassungsgerichts geführt. ${ }^{7}$ Die verfassungsrechtliche Sichtweise des Gerichts ist zudem durch die Normsetzung und den Normbezug des Grundgesetzes geprägt. Die vergleichende Wahlsystemforschung kann sich natürlich diese Anbindung nicht zu eigen machen. Zwar weisen demokratische Verfassungen überall auf der Welt aus, dass die Wahlen allgemein, gleich, direkt und geheim zu sein haben. Die Wahlsysteme aber sind in den Ländern höchst verschieden, auch und gerade im Bereich der Verhältniswahlsysteme. Fast nirgends wird aus den Wahlrechtsgrundsätzen wie von Seiten des Bundesverfassungsgerichts abgeleitet, dass sich Verhältniswahlsysteme verfassungsrechtlich nicht nur am gleichen Zählwert, sondern auch und gerade am gleichen Erfolgswert der Wählerstimmen messen lassen müssen. Vor dem Hintergrund der international vergleichenden Wahlsystemforschung verengt und verzerrt die bundesrepublikanische Rechtsprechung zum Wahlsystem den Bewertungshorizont von Wahlsystemen, was sich in folgenden Mängeln ausdrückt:

Erstens fehlt es an empirischem Bezug. Dies wird darin deutlich, dass Verhaltensvariablen, die die Auswirkungen von Wahlsystemen mitbestimmen, nicht ausreichend berücksichtigt werden. Institutionen gestalten Wählerverhalten mit. Wahlsysteme etablieren grundsätzlich unterschiedliche Bedingungen dafür, dass die Stimme eines Wählers für etwas zählt. Dass bestimmte Regelungen eines Wahlsystems Bürger in ihrer Stimmabgabe beeinflussen ${ }^{8}$, ist ein gewollter Effekt von Wahlsystemen. Verhältniswahlsysteme bilden da keine Ausnahme.

7 Vgl. etwa Jochen Abr. Frowein, Die Rechtsprechung des Bundesverfassungsgerichts zum Wahlrecht, in: AöR 1974, S. 72 - 110. Siehe auch Michael Wild, Die Gleichheit der Wahl. Dogmengeschichtliche und systematische Darstellung, Berlin 2003.

8 Vgl. Gary W. Cox, Making Votes Count. Strategic Coordination in the World's Electoral Systems, Cambridge 1997; Franz U. Pappi / Susumu Shikano, Wahl und Wählerforschung, Baden-Baden 2007; Pippa Norris, Electoral Engineering. Voting Rules and Political Behavior, Cambridge 2008. 
Zweitens werden keine international komparativen Erfahrungen herangezogen, die Grundlage der konzeptionellen und politikberaterischen Weiterentwicklung der Wahlsystemlehre sind. Der Blick nur auf die deutschen Gegebenheiten und Erfahrungen engt die normativen Vorstellungen und funktionalen Gestaltungsmöglichkeiten von Wahlsystemen unnötig ein. Heute entscheidet der Gesetzgeber nicht mehr zwischen den klassischen Wahlsystemen von Mehrheitswahl und Verhältniswahl, sondern gestaltet Wahlsysteme entsprechend den funktionalen Anforderungen, die in spezifischen Kontexten an sie gestellt werden.

Drittens mangelt es an umfassenderen Bewertungsmaßstäben für Wahlsysteme, was in deren eindimensionaler Zuspitzung und disparaten Anwendung je nachdem, ob es sich um Verhältniswahl oder Mehrheitswahl handelt, deutlich wird. Es geht bei Wahlsystemen nicht nur um unmittelbare Auswirkungen auf das Verhältnis von Stimmen und Mandaten, sondern um weiter reichende Wirkungen, auch um Nebenwirkungen und Folgekosten in anderen als etwa durch die Wahlrechtsgleichheit thematisierten Bezügen. Und es empfehlen sich auch Maßstäbe, die zugleich auf Mehrheitswahl und auf Verhältniswahl angewandt werden können, um einen sinnvollen Vergleich zu ermöglichen.

\section{Der Grundsatz von Mehrheitswahl und Verhältniswahl}

Folgt man dem Urteilsspruch, so entspricht dem „Zweck der Mehrheitswahl (...), dass nur die für den Mehrheitskandidaten abgegebenen Stimmen zur Mandatszuteilung führen. Die auf den Minderheitskandidaten abgegebenen Stimmen bleiben hingegen bei der Vergabe der Mandate unberücksichtigt“. „Ziel der Verhältniswahl“, so meint das Gericht, „ist es, dass alle Parteien in einem möglichst den Stimmenzahlen angenäherten Verhältnis in dem zu wählenden Organ vertreten sind“. In diesen Bestimmungen wird ein Vergleich asymmetrischer Natur vorgenommen. Mehrheitswahl wird nach dem Entscheidungsprinzip im Wahlkreis, Verhältniswahl nach der politischen Repräsentation im Parlament definiert. Diese schiefe Gegenüberstellung ist jedoch in der Wahlsystemlehre längst überwunden.?

Wenn Roman Herzog sich für die relative Mehrheitswahl ausspricht ${ }^{10}$, dann meint er doch nicht primär, dass in den Wahlkreisen die relative Mehrheit der Stimmen entscheiden soll. Das tut sie ja jetzt bereits für die Hälfte der Abgeordneten. Er hat die Auswirkungen, die dieses Wahlsystem auf die Zusammensetzung des Parlaments ausübt, im Blick. Paul Kirchhof, ebenfalls ehemaliger Verfassungsrichter, ist in Zielorientierung von Mehrheitswahl auf die Mehrheitsverhältnisse im Parlament noch expliziter, wenn er vorschlägt ${ }^{11}$, dass diejenige Partei oder Koalition, die die relative Mehrheit der Stimmen erringt, automatisch mit der absoluten Mehrheit der Mandate ausgestattet werden soll - Quintessenz der Reform Silvio Berlusconis in Italien. ${ }^{12}$ Das BVerfG hat die Unterscheidung von Entscheidungsregel und Repräsentationsprinzip nicht nachvollzogen. Diese Unterscheidung ist aber wich-

9 Dieter Nohlen, Begriffliche Einführung in die Wahlsystematik, in: Dolf Sternberger / Bernhard Vogel (Hrsg.), Die Wahl der Parlamente und anderer Staatsorgane, Bd. 1: Europa, Berlin 1969, S. 39; ders., Wahlsysteme der Welt, München 1978, S. 48 ff.; Giovanni Sartori, Comparative Constitutional Engineering. An Inquiry into Structures, Incentives, and Outcomes, Houndmills u.a. 1994, S. 4.

10 Vgl. SZ vom 17. März 2008.

11 Vgl. Alpbacher Gespräche, Alpbach News vom 27. August 2008.

12 Vgl. Klaus Poier, Minderheitenfreundliches Mehrheitswahlrecht, Wien u.a. 2001. 
tig, wenn es beispielsweise die Frage der Gewährleistung von Erfolgswertgleicheit der Wählerstimmen zu bewerten gilt, was später noch thematisiert wird. Definiert man Mehrheitswahl von der technischen Seite her, so ist Erfolgswertgleichheit ausgeschlossen. Die Stimmen für die Minderheit(en) fallen unter den Tisch. Versteht man Mehrheitswahl als Repräsentationsprinzip, so ist es durchaus möglich, Mehrheitswahlsysteme zu gestalten, die den Gesichtspunkt der Erfolgswertgleichheit in gewisser Weise mitberücksichtigen. Jedes Mehrheitswahlsystem, das mit Wahlkreisen operiert, in denen mehr als ein Abgeordneter zu wählen ist, eröffnet bereits eine anteilige Vergabe von Mandaten. Im Dreierwahlkreissystem kann je nach Struktur des Parteiensystems bereits eine beachtliche Erfolgswertgleichheit der Stimmen erzielt werden. Geradzahlige Wahlkreisgrößen sind besonders geeignet, den Minderheitsstimmen einen überproportionalen Erfolgswert einzuräumen. Hinsichtlich der Verhältniswahl schließt ihre Definition als Entscheidungsmaßstab die Verletzung der Erfolgswertgleichheit der Stimmen eigentlich aus. Es liegen nur mathematische Begrenzungen vor, besonders in kleinen Wahlkreisen. Hingegen lassen sich für Verhältniswahl, verstanden als Repräsentationsprinzip, durchaus intendierte Einschränkungen der Erfolgswertgleichheit denken, da diese Zielsetzung in Konkurrenz zu anderen tritt, die gesamtpolitisch und anhand übergeordneter Kriterien (Funktions- und Überlebensfähigkeit der Demokratie) hinzutreten. Das mag sich etwa (als dem einfachsten Beispiel) in der Etablierung von Sperrklauseln niederschlagen.

Die politische Zielvorstellung der Mehrheitswahl besteht darin, die parlamentarische Mehrheit für eine Partei oder ein Parteienbündnis herzustellen. Dabei geht es im Wesentlichen darum, eine Partei (ein Parteienbündnis), die (das) in Stimmen nicht die absolute Mehrheit erhalten hat, nach Mandaten zur parlamentarischen Mehrheit zu befähigen. So räumt auch Hans Meyer, auf die Wahlsystemlehre von Dieter Nohlen verweisend, ein: Der Zweck der Mehrheitswahl „liegt heute nicht primär darin, die (relative) Wählermehrheit einer Partei in einem Wahlkreis in ein Mandat umzusetzen, sondern darin, die Mandatsmehrheit einer Partei im Parlament zu ermöglichen, und zwar gerade dann, wenn keine Wählermehrheit vorhanden ist"13. Hingegen sollen in Verhältniswahlsystemen im Prinzip die in der Bevölkerung bestehenden sozialen Kräfte und politischen Gruppen möglichst getreu wiedergegeben werden. Stimmenanteile und Mandatsanteile sollen sich in etwa entsprechen. Als weiteres Ziel der Verhältniswahl wird gemeinhin verstanden, dass auch Zählwert und Erfolgswert der Stimmen der Wähler sich in etwa entsprechen. Historisch und systematisch ist dies eine Folge der angestrebten Proportionalität von Stimmen und Mandaten, nicht ihre Voraussetzung ${ }^{14}$, und hat sich erst im Rahmen der allgemeinen Entwicklung der Gleichheitsidee auch im Felde der Wahlsysteme eine gewisse, in Deutschland eine außerordentliche Anerkennung verschafft.

Diese Zielorientierung der Verhältniswahl bedeutet aber nicht, dass bei ihr „alle Parteien in einem möglichst den Stimmenzahlen angenäherten Verhältnis in dem zu wählenden Organ vertreten sind" 15 , wie es im hier kommentierten Urteil heißt. Auch bei der Verhältniswahl bestehen Repräsentationshürden, etwa bei der geläufigsten Form, der Verhältniswahl in Wahlkreisen unterschiedlicher Größe. Sie verhindern legitimerweise, dass alle Par-

13 Hans Meyer, Demokratische Wahl und Wahlsystem, in: Josef Isensee / Paul Kirchhof (Hrsg.), Handbuch des Staatsrechts der Bundesrepublik Deutschland, Bd. 2, Heidelberg 1987, S. 261.

14 Vgl. Karl Braunias, Das parlamentarische Wahlrecht, Bd. 2, Berlin / Leipzig 1932, S. 191 - 203.

15 BVerfG 2 BvC 1/07 und 2 BvC 7/07, a.a.O. (Fn. 1), Rn. 93. 
teien in ein Parlament einziehen. Die Größe der Wahlkreise ist - neben der gesetzlichen Sperrklausel - der wichtigste Faktor für Disproportionalität der Wahlergebnisse. ${ }^{16}$ Der monierte Satz ist Ausdruck der Vorstellung, dass bei Verhältniswahl nicht nur der Zählwert, sondern auch der Erfolgswert der Stimmen gleich zu sein habe. Diese Vorstellung durchzieht das gesamte Urteil und wird auf die Spitze getrieben, wenn es um die Frage des Verrechnungsverfahrens geht, das 2008 reformiert wurde (statt Hare/Niemeyer nun SainteLaguë(Schepers). Als „ungleiche Ergebnisse“ betrachtet das BVerfG alles, was „nicht exakt verhältnismäßig und daher auch nicht exakt dem Grundsatz der Erfolgswertgleichheit“ entspricht, davon ausgenommen wird nur die „unausweichliche Folge eines jeglichen Verteilungsverfahrens (als) gerechtfertigt angesehen "17. Der Erfolgswert wird damit an maximaler Proportionalität der Wahlergebnisse festgemacht. Mit solchen Vorstellungen wird zum Wahlsystem der Weimarer Republik zurückgekehrt, wo es im automatischen System der Verhältniswahl für eine bestimmte Zahl von Stimmen im gesamten Reichsgebiet ein Mandat gab. Es besteht aber kein Zweifel, dass die personalisierte Verhältniswahl in dem damals politisch durchsetzbaren Umfang als alternatives Wahlsystem zur reinen Verhältniswahl betrachtet wurde. ${ }^{18}$ Das hat sich auch in der zwar fehlerhaften, aber immer noch lebendigen Vorstellung manifestiert, in der personalisierten Verhältniswahl werde die eine Hälfte der Mandate nach Mehrheitswahl, die andere nach Verhältniswahl vergeben.

In Kategorien der Wahlsystematik gedacht, könnte man weiterhin aus dem Urteil die Vorstellung eines unipolaren Kontinuums der Wahlsysteme herauslesen, derzufolge die wesentliche Differenzierung zwischen der reinen Verhältniswahl und allen anderen Wahlsystemen liegt: hier die „denkbar genaueste Proportion zwischen den erreichten Stimmengewinnen und dem Anteil an Parlamentssitzen“, dort „eine zunehmende Restriktion gegenüber den Chancen der jeweils kleineren Parteien (...) bis zu einem Zustand, der praktisch nur noch zwei Parteien die Chance lässt, Parlamentssitze zu gewinnen" 19 . Verhältniswahl in Form der „denkbar genauesten Proportion“ als Kern der Wahlrechtsgleichheit und Wahlsystembewertung ist „Weimar“.

Aus vergleichender wahlsystematischer Sicht muss die grundsätzliche Frage gestellt werden, wie sinnvoll der absolut gesetzte Vorrang von Exaktheit im Proporz bei der personalisierten Verhältniswahl ist. Diese ist nur eine Dimension von Sinnhaftigkeit, andere sind Einfachheit, Transparenz und Nachhaltigkeit. Genauigkeit in der proportionalen Verteilung wird in der Regel mit einem höheren Grad an Komplexität in der proportionalen Berechnung erkauft. Es fragt sich, wer das allein auf Exaktheit abhebende Verfahren noch verstehen kann. Der einfache Wähler sicherlich nicht. Damit geht viel an Transparenz verloren. Nachhaltigkeit kann die größere Genauigkeit für sich auch nicht verbuchen, weil die Kritik an der personalisierten Verhältniswahl wegen der unverständlichen Berechnung der Sitze

16 Vgl. Arend Lijphart, Electoral Systems and Party Systems, Oxford 1994.

17 BVerfG 2 BvC 1/07 und 2 BvC 7/07, a.a.O. (Fn. 1), Rn. 104. Demgegenüber stellte Hans Meyer bereits 1978 treffend fest: „Es sind ebenso mehr oder weniger proportionale Systeme denkbar wie solche mit mehr oder weniger mehrheitsbildender Wirkung." Hans Meyer, Wahlsystem und Verfassungsordnung. Bedeutung und Grenzen wahlsystematischer Gestaltung nach dem Grundgesetz, Frankfurt am Main 1973, S. 189.

18 Friedrich Karl Fomme, Von der Weimarer Verfassung zum Bonner Grundgesetz, Tübingen 1962, S. 162 f.; Bernhard Vogel / Dieter Nohlen / Rainer-Olaf Schultze, Wahlen in Deutschland, Berlin / New York 1971, S. 188.

19 Hans Meyer, a.a.O. (Fn. 17), S. 189. 
und die Forderung nach einem grundlegenden Wahlsystemwechsel zunehmen dürften. Diesen Schritt hat jüngst der ehemalige Verfassungsrichter Hans Hugo Klein vollzogen. Aus dem Recht des Bürgers heraus, „das Wahlrecht, auf dessen Grundlage er sein Parlament wählt, ohne Inanspruchnahme von Wahlrechtsexperten und Mathematikern verstehen zu können“, erwägt er eine Option, „das jedermann leicht verständliche Mehrheitswahlrecht einzuführen" 20 .

\section{Personalisierte Verhältniswahl als Wahlsystemtyp}

Das Bundesverfassungsgericht hat sich immer damit schwer getan, das Wahlsystem zum Bundestag zu bestimmen und zu klassifizieren. Symptomatisch ist das Urteil vom 10. April $1997^{21}$, mit dem damals die Überhangmandate ohne Ausgleich und Verrechnung als mit dem Grundgesetz vereinbar erklärt wurden. Vier zu vier lautete das Abstimmungsergebnis. Die eine Gruppe von Verfassungsrichtern verwendete viele Beschreibungen darauf, wie die personalisierte Verhältniswahl zu verstehen sei, angefangen vom Bild der Kombination von Mehrheitswahl und Verhältniswahl über dasjenige des Verhältnisausgleichs bis hin zu jenem einer der Verhältniswahl „vorgeschalteten Mehrheitswahl“22. Der Proporz nach Zweitstimmen sei vom Gesetzgeber nicht zum ausschließlichen Verteilungskriterium erhoben worden, auch wenn die Bundestagswahl den Grundcharakter einer Verhältniswahl trage. Demgegenüber begriff die unterlegene Gruppe von Verfassungsrichtern, definitorisch in sich schlüssig, das bundesrepublikanische Wahlsystem als Verhältniswahl, allerdings unter Verkennung der Kombinationsproblematik und unter ausschließlicher Orientierung des Systems am Proporz, so als sei die verhältnismäßige Verteilung der Mandate der einzige Maßstab.

Nach $\$ 1$ Abs. 1 Satz 2 Bundeswahlgesetz wird nach den Grundsätzen einer mit der Personenwahl verbundenen Verhältniswahl gewählt. Von Mehrheitswahl ist gar nicht die Rede. Erst bei der Festlegung des Entscheidungsmaßstabes bei der „Wahl in den Wahlkreisen“ ( $\$ 5$ Satz 2: „Gewählt ist der Bewerber, der die meisten Stimmen auf sich vereinigt.“) wird vermittelt, dass nach relativer Mehrheitswahl gewählt wird. Der Begriff „personalisierte" Verhältniswahl setzt den Akzent noch klarer: Es liegt Verhältniswahl vor. Der Einbau von Elementen der Personenwahl ändert daran nicht das Geringste, weshalb alle Definitionen, die das deutsche Wahlsystem als Mischsystem bezeichnen, in die Irre führen. Verhältniswahl als Repräsentationsprinzip für die Wahl zum Bundestag insgesamt wird mit dem Entscheidungsmaßstab der Mehrheitswahl zwecks Personenwahl im Falle der Hälfte der Mitglieder des Bundestags kombiniert. Mit dieser Verbindung können Einschränkungen des Proporzprinzips einhergehen, dessen uneingeschränkte Anwendung ohnehin durch die Einführung einer Sperrklausel vom Gesetzgeber nicht gewollt wurde.

20 Hans Hugo Klein, Eine klare Wahl, in: FAZ vom 14. August 2008, S. 7.

21 BVerfG 2 BvC 3/96, a.a.O. (Fn. 3).

$22 \mathrm{Im}$ englischsprachigen Schrifttum wird leider stets von einem Mischsystem gesprochen. Belege in Dieter Noblen, Wahlrecht und Parteiensystem, Opladen 2009, S. 280 - 284. Auch in Deutschland lässt die irrige Bezeichnung nicht nach, so jüngst bei Kommentierung desselben Urteils in der FAZ vom 4. Juli 2008, S. 2. 
Im hier kommentierten Urteil setzt sich die Unsicherheit des Gerichts in der Definition des deutschen Wahlsystems fort, indem es an einer Stelle heißt, es finde ein „Verhältnisausgleich durch die nach Verhältniswahl vergebenen Mandate" 23 statt. An anderer Stelle hingegen wird betont, „dass grundsätzlich die Erststimme nur darüber entscheidet, welche Personen als Wahlkreisabgeordnete in den Bundestag einziehen, dass sich die Mehrheitsverhältnisse aber - im Grundsatz - allein nach dem Zweitstimmenverhältnis richten " 24 . Die definitorischen Unterschiede scheinen unerheblich, zumal unter mathematischen Gesichtspunkten gänzlich irrelevant. Sie sind es aber empirisch nicht. Verhältniswahlsysteme mit Ausgleichsmandaten, so genannte kompensatorische Wahlsysteme, streben eine proportionale Verteilung an, erreichen sie aber häufig nicht, da viele andere intervenierende Variablen dies verhindern können. Auch können in der technischen Gestaltung Akzente gesetzt werden, die anderen als Proporzgesichtspunkten folgen. Das ist beispielsweise bei der Verhältniswahl in Ungarn der Fall, wo zunächst 176 Mandate in Einerwahlkreisen nach absoluter Mehrheit mit Stichwahl vergeben werden. Die entstandenen Disproportionen werden dann in zwei weiteren Stufen der Vergabe von insgesamt 210 Mandaten zu korrigieren versucht. Eine der Systemintention entsprechende hohe Proportionalität der Wahlergebnisse wurde jedoch aufgrund entgegen wirkender Verhaltensmuster der Parteien und der Wählerschaft nicht erreicht. ${ }^{25}$ Bei der personalisierten Verhältniswahl hingegen ist durch die Anwendung der proportionalen Entscheidungsregel auf nationaler Ebene die verhältnismäßige Verteilung der Mandate an die Parteien auf jeden Fall gewährleistet. Die Definition der Wahlsysteme ist ohne Zweifel von Belang für die Frage, welche Bedeutung bestimmten normativen Kriterien in deren Bewertung beizumessen ist. ${ }^{26}$

\section{Der Grundsatz der Gleichheit der Wahl}

Es ist hier nicht der Ort, die Geschichte der höchstrichterlichen Anwendung des Grundsatzes der Gleichheit bezogen auf einzelne Elemente der personalisierten Verhältniswahl, darunter der Überhangmandate, nachzuzeichnen. ${ }^{27}$ Hier soll es nur um die zentralen Argumente des Bundesverfassungsgerichts im Zusammenhang mit dem negativen Stimmgewicht gehen. Sie ranken um die Begriffe Gleichheit, Erfolgswertgleichheit und Erfolgschancengleichheit. Der Grundsatz der Gleichheit der Wahl sei verletzt, weil er fordert, dass der Erfolgswert jeder Stimme gleich ist. Das bedeute, dass die Stimme für die Partei, für die sie abgegeben wurde, „eine positive Wirkung entfalten können muss“28. Auch die Erfolgschancengleichheit sei beeinträchtigt, wenn mit der Abgabe einer Stimme die Chance besteht, dem eigenen Wahlziel zu schaden.

23 BVerfG 2 BvC 1/07 und 2 BvC 7/07, a.a.O. (Fn. 1), Rn. 27.

24 Ebenda, Rn. 35.

25 Vgl. Dieter Nohlen, a.a.O. (Fn. 22), S. 387 - 393. Als weiteres Beispiel könnte Italien nach der Reform von 1993 dienen, vor allem hinsichtlich der Wirkung von Kontextfaktoren. Vgl. ebenda, S. $222-228$.

26 Vgl. Hans Meyer, a.a.O. (Fn. 17), S. 182.

27 Vgl. Dieter Nohlen / Nicolas Nohlen, El sistema electoral alemán y el Tribunal Constitucional Federal, La igualdad electoral a debatre - con una mirada a Venezuela, in: Revista de Derecho Público 2007, Nr. 109, S. 7 - 26.

28 BVerfG 2 BvC 1/07 und 2 BvC 7/07, a.a.O. (Fn. 1), Rn. 103. 
Die Wahlsysteme unterscheiden sich gerade darin, dass sie den Erfolgswert der Stimmen differenzieren. Das hat auch das BVerfG bereits in den Anfängen seiner Rechtsprechung zum Wahlsystem zum Ausdruck gebracht und die Erfolgswertgleichheit sogar vom Wahlsystem abhängig gemacht. Sie gilt danach für die Verhältniswahl als Maßstab der Wahlrechtsgleichheit, nicht aber für die Mehrheitswahl (wobei im Gedächtnis zu halten ist, dass das Gericht bei Mehrheitswahl an die Entscheidungsregel denkt und nicht an das Repräsentationsprinzip). Das Gericht hat zugleich mit dieser starren Unterscheidung die Verhältniswahl dem strikten Kriterium unterworfen, ein Maximum an Erfolgswertgleichheit der Stimmen zu gewährleisten. Abweichungen von der Wahlrechtsgleichheit sind seither zu begründen. Das hat im System der personalisierten Verhältniswahl für die Sperrklausel, die Grundmandatsklausel, die Überhangmandate und das Verrechnungsverfahren gegolten, die allesamt höchstrichterlich auf ihre Verfassungskonformität überprüft worden sind und im Prinzip für mit dem Gleichheitsgrundsatz vereinbar gehalten wurden.

Der Ansatz der vergleichenden Wahlsystemforschung ist ein anderer. Verhältniswahl und Mehrheitswahl (beide definiert als Repräsentationsprinzipien) variieren intern im Erfolgswert der Stimmen. Verhältniswahlsysteme streben Erfolgswertgleichheit in höherem Maße an als Mehrheitswahlsysteme. Aber es ist weder so, dass Verhältniswahlsysteme der möglichst großen Annäherung beider Werte verpflichtet sind, noch dass diese durch das höchste $\mathrm{Maß}$ an formaler proportionaler Prinzipientreue am ehesten gewährleistet werden könnte. Empirische Gegebenheiten sind zu berücksichtigen. Der Grad der Gleichheit von Zählwert und Erfolgswert der Stimmen ist nämlich nicht nur von der Ausgestaltung des Wahlsystems, sondern vom Parteiensystem und vom Wählerverhalten abhängig. Dabei gilt: Je mehr Parteien (möglicherweise als Folge von Verhältniswahl), desto geringer die Erfolgswertgleichheit. Auch der Wähler reagiert auf das Wahlsystem - und er kann sich dabei verkalkulieren. Dabei gilt: Je mehr Proporz in ein Wahlsystem eingebaut ist, desto eher ist der Wähler geneigt anzunehmen, dass seine Stimme zählt, auch für kleine und kleinste Parteien. Er unterschätzt die Repräsentationshürden, die nach wie vor rein mathematisch durch die beschränkte Zahl der zur Verfügung stehenden Mandate gegeben sind, weshalb einer großen Zahl von Stimmen kein Erfolg beschieden sein kann. Die Stimmen, die auf Kandidaten oder Parteien entfielen, die nicht erfolgreich waren, lassen die faktische Erfolgswertgleichheit sinken. Wird der Wähler hingegen durch effektive Hürden, die die Erfolgswertgleichheit erkennbar einschränken, angehalten, „nützlich“ zu stimmen, also seine Stimme Parteien oder Kandidaten zu geben, die Aussicht haben, gewählt zu werden, so reduziert sich die Zahl der Stimmen, die ohne Erfolg bleiben. Die faktische Erfolgswertgleichheit der Stimmen steigt. Diese zur jeweiligen Absicht gegenläufigen Auswirkungen, die reine und mit Sperrklauseln versehene Verhältniswahlsysteme haben, nenne ich das Proporzparadox.

Es ist also möglich, dass bei Ausstattung eines Wahlsystems mit mehr theoretischer Erfolgswertgleichheit weniger effektive Erfolgswertgleichheit herauskommt. Generell kann ab einem bestimmten Maß an Realisierung der Idee, wahlrechtlich Zählwert und Erfolgswert von Stimmen anzugleichen, gelten: Je mehr Proporz, desto weniger Erfolgswertgleichheit. Daher ist das Argument der Erfolgswertgleichheit für die Bewertung von Verhältniswahlsystemen nur beschränkt tauglich. Es kann sich empirisch selbst aushebeln. Aus diesem Grund ist es auch nicht sinnvoll zu argumentieren, der Grundsatz der gleichen Wahl sei verletzt, weil er fordere, dass der Erfolgswert jeder Stimme gleich sei. Schon gar nicht ist einsichtig, dass der Grundsatz der Gleichheit der Wahl, an der Erfolgswertgleichheit festge- 
macht, bedeute, dass die Stimme, die für eine Partei abgegeben wurde, „eine positive Wirkung entfalten können muss“. Sinnvoll ist allein die Forderung, dass auszuschließen ist, dass die Stimmabgabe für eine Partei eine negative Wirkung, also eine solche zu ihren Lasten entfaltet.

Letztlich gilt es also rechtspolitisch auszuschließen, dass in einem Wahlsystem bei der Abgabe einer Stimme die Gefahr besteht, dass sie gegen die Partei wirken kann, der sie zugute kommen sollte, allgemeiner formuliert, dass sie gegen das Ziel wirkt, das man mit ihr anstrebt. Das Problem ist dabei nicht, dass Wahlsysteme die Wähler anregen, sich wahltaktisch zu verhalten, um ihren politischen Willen, der parlamentarischen Vertretung, dem Wahlsieg einer Partei oder dem Zustandekommen einer bestimmten Regierungskoalition zum Durchbruch zu verhelfen. Es genügt, dass der Wähler den Erfolgsmechanismus eines Wahlsystems erkennen und sich dementsprechend rational verhalten kann. Insofern ist das Wahlverhalten vieler CDU-Wähler bei der Nachwahl in Dresden, das den Anstoß für die Befassung des Gerichts mit dem ungleichen Stimmgewicht gegeben hat, durchaus legitim und spricht für deren Intelligenz. Sie wussten das System zu handhaben, um kein Mandat einzubüßen. Die Nachwahl in Dresden hat vielmehr Licht auf das Problem geworfen, dass der Wähler normalerweise bei der Verrechnung der Direktmandate auf Landesebene nicht einschätzen kann, ob seine Stimme für eine Partei auch dieser zugute kommt, ihren Mandatsanteil zu stärken hilft oder das Gegenteil bewirkt. Es ist der Mangel an Transparenz, der die Wählerstimme willkürlichen Effekten aussetzt. Der Wähler kann sich nicht rational verhalten. Entscheidend ist demnach, nicht das taktische Wahlverhalten als dem Kern der Wahl fremd oder der Gleichheit der Wahl zuwider laufend zu betrachten, sondern die Intransparenz des Wirkungsmechanismus in der Verbindung einzelner Elemente des Wahlsystems.

Diese Differenzierung ist insofern wichtig, als ohne sie der argumentative Boden dafür bereitet wird, andere Mechanismen des Wahlsystems, die wahltaktisches Verhalten ermöglichen und fördern, verfassungsrechtlich zu hinterfragen. Zu denken ist an das Zweistimmensystem der personalisierten Verhältniswahl, das ja auch schon eine Verfassungsbeschwerde ausgelöst hat.

Generell sind Überlegungen, die in die Richtung gehen, Wahlsysteme an der strikt verfolgten Idee der Erfolgswertgleichheit zu messen und eine möglichst große Annäherung von Zählwert- und Erfolgswertgleichheit zu fordern, eindimensional: Sie vernachlässigen die Nebenwirkungen und möglichen Folgekosten; sie verletzen das Gebot, dass Wahlsysteme mehr als einer einzigen Zielvorstellung gerecht zu werden haben. Ohne Zweifel ist bei Verhältniswahl Proportionalität der Ergebnisse hochrangig anzusetzen, bedeutend höher als bei Mehrheitswahl - aber eine einseitige Berücksichtigung rechtfertigt sich nicht.

Das BVerfG schiebt in seinem Urteil noch das Argument nach, dass auch die Erfolgschancengleichheit durch das negative Stimmgewicht beeinträchtigt sei, wenn mit der Abgabe einer Stimme die Gefahr bestehe, dem eigenen Wahlziel zu schaden. Gewiss relativiert die Erfolgschancengleichheit den Grundsatz der Erfolgswertgleichheit, ähnlich wie Chancengleichheit den Grundsatz der Gleichheit relativiert. Damit will das Gericht vielleicht zum Ausdruck bringen, dass auch bei geringeren Gleichheitsansprüchen an ein Wahlsystem eine zur Wahlintention negative Auswirkung der Wählerstimme nicht hingenommen werden kann. Aber auch die Erfolgschancen, die Wähler haben, sind nach Wahlsystemen unterschiedlich, auch und gerade in Verhältniswahlsystemen: Während bei der klassischen relativen Mehrheitswahl generell gilt, dass die Erfolgschancen für die Wähler kleinerer 
landesweiter Parteien als Folge der Anwendung der Entscheidungsregel der Mehrheitswahl in Einerwahlkreisen im Prinzip gleich null sind, sind sie bei Verhältniswahlsystemen nicht nur zwischen diesen, sondern auch innerhalb einzelner Systeme höchst unterschiedlich. In Verhältniswahlsystemen mit Wahlkreisen unterschiedlicher Größe ist die Erfolgschancengleichheit der Wählerstimmen überhaupt nicht gegeben. In großen Wahlkreisen sind die Erfolgschancen für Wähler kleiner Parteien tendenziell vorhanden, in kleinen Wahlkreisen eher nicht. Empirisch ist hier im Einzelfall die geographische Verteilung der Wählerpräferenzen eine wichtige Variable.

\section{Der Grundsatz der Unmittelbarkeit der Wahl}

Das BVerfG sieht auch den Grundsatz der Unmittelbarkeit der Wahl verletzt. Erneut wird hier mit der positiven oder negativen Auswirkung der Stimme argumentiert und gemeint, der Grundsatz sei verletzt, weil der Wähler nicht erkennen könne, wie sich seine Stimme auswirke.

Die Wahlen zum Bundestag, so sagt es Art. 38 GG, sind direkt. Der Grundsatz der Unmittelbarkeit bezieht sich darauf, dass zwischen Wähler und zu bildender Repräsentation kein Gremium zwischengeschaltet sein darf. Der Hinweis auf das Verfahren zur Wahl des amerikanischen Präsidenten mag genügen, um ex negativo klar zu machen, was mit unmittelbar gemeint ist. In den USA bestimmen bei Präsidentschaftswahlen die Wähler in den einzelnen Bundesstaaten in Urwahlen die Wahlmänner, die dann den Präsidenten wählen. Diese Wahlen sind indirekt. Dabei kann es vorkommen, dass ein gewählter Präsident die Stimmen der Mehrheit der Wahlmänner auf sich vereint, obwohl nicht die Mehrheit der Wählerstimmen auf ihn entfallen ist. Im Prinzip sind die Wahlmänner der Bundesstaaten an die Mehrheitsentscheidung der Wähler gebunden. Es hat aber auch schon Fälle gegeben, in denen die Wahlmänner sich anders verhielten. Die indirekte Wahl hat also Tücken. Auf jeden Fall wird der Wählerwille mediatisiert, was die direkte Wahl ausschließt.

Nun wird im Urteil argumentiert, dass auch der Grundsatz der direkten Wahl durch das negative Stimmgewicht verletzt sei. Der Vertreter der Wahlprüfungsbeschwerde argumentierte, „weil die Stimmen nicht direkt wirkten, sondern die Anhänger gezwungen seien, ihrer Partei die Stimme zu verweigern "29. Es braucht nicht weiter erläutert zu werden, dass diese Begründung mit dem eigentlichen Gegenstand, der mit der Unmittelbarkeit der Wahl normiert wird, nichts zu tun hat. Darüber hinaus tritt der beschriebene „Zwang “ ja nicht bei einer normalen Wahl auf, da, wie schon erwähnt, der Wähler nicht weiß, wie seine Stimme wirkt, er sich folglich auch zu nichts gezwungen sehen kann. Das negative Stimmgewicht ist, wie im Urteil richtig festgestellt wird, „nicht vorhersehbar und nicht planbar und von dem einzelnen Wähler kaum zu beeinflussen “30. Weiß er aber Bescheid, wie bei einer Nachwahl, wenn entsprechende Berechnungen vorliegen, die Wahlchancen seiner Partei liegen, so kann er sich wahltaktisch so verhalten, dass seiner Partei der größte Nutzen entsteht. Warum soll rationales Wahlverhalten nicht unmittelbare Wahl sein? Das Gericht hat sich der Argumentation des Beschwerdeführers nicht angeschlossen, aber dennoch die Verletzung der Unmittelbarkeit der Wahl ins Urteil geschrieben. Der Grundsatz fordere

29 BVerfG 2 BvC $1 / 07$ und 2 BvC 7/07, a.a.O. (Fn. 1), Rn. 50.

30 Ebenda, Rn. 102. 
„ein Wahlverfahren, in dem der Wähler vor dem Wahlakt erkennen kann, welche Personen sich um ein Abgeordnetenmandat bewerben und wie sich die eigene Stimmabgabe auf Erfolg oder Misserfolg der Bewerber auswirken kann. Nicht entscheidend sei, dass die Stimme tatsächlich die vom Wähler beabsichtigte Wirkung entfaltet, ausreichend ist die Möglichkeit einer positiven Beeinflussung des Wahlergebnisses"31, womit wohl gemeint ist, eine Beeinflussung in dem vom Wähler intendierten Sinne. In der Tat gehört zur Wahl der Wahlvorschlag 32 , ist also ein Erfordernis an die Wahl selbst, nicht erst ihrer Unmittelbarkeit. Der weitere Gesichtspunkt betrifft die Transparenz für den Wähler: Die Frage, „wie sich die eigene Stimmabgabe auf Erfolg oder Misserfolg der Bewerber auswirken kann" ${ }^{33}$, hat im Grunde auch nichts mit der Unmittelbarkeit zu tun. Im Ergebnis zeigt sich wenig Substanz für die Feststellung, dass der Grundsatz der Unmittelbarkeit der Wahl vom negativen Stimmgewicht betroffen sei.

Ähnlich verhält es sich mit der Verletzung der Freiheit der Wahl. Sie liege, so der Beschwerdeführer, vor, „weil die Wähler, die ihrer Partei mit ihrer Stimme schaden könnten, davon abgehalten würden, dieser Partei ihre Stimme zu geben "34. Es ist wichtig, dieser Begründung zu widersprechen, weil andernfalls zukünftig argumentiert werden könnte, dass ein Wähler, der rational wählt, also Schaden von seiner Partei abwendet durch welches Wahlverhalten auch immer, gegen die Freiheit der Wahl verstößt. Erfreulicherweise hat sich das Gericht, allerdings ohne große Prüfung, die Sicht des Beschwerdeführers nicht zu eigen gemacht.

Es fragt sich zudem, ob es der richterlichen Argumentation im Zusammenhang mit der Verletzung der Unmittelbarkeit der Wahl, die sich an den positiven oder negativen Auswirkungen der Stimme festmacht, überhaupt bedurft hätte. Denn die Problematik der Unsicherheit, welchen Effekt die Stimme hat, stellt sich ja nicht mehr, wenn die Stimme mit negativer Auswirkung auf die gewählte Partei nicht mehr zulässig ist. Den Grundsatz der Unmittelbarkeit der Wahl zu bemühen, ist nämlich nicht ganz unproblematisch. Erneut gilt es zu bedenken, dass in etlichen Wahlsystemen der Wähler bei der Stimmabgabe nicht genau erkennen kann, was mit seiner Stimme geschieht. Bei Mehrheitswahlsystemen ist dies häufig für ihn überschaubar. Wie sollte er aber bei Verhältniswahlsystemen beispielsweise das Verrechnungsverfahren im Kopf haben, unter dessen Anwendung auch seine Stimme zur Ermittlung der Wahlergebnisse beiträgt, oder mehrere Verrechnungsebenen, auf denen seine Stimme möglicherweise zählt. Die Unmittelbarkeit der Wahl darf durch diese natürliche Ignoranz nicht in Frage gestellt sein. Es wäre auch hier angebracht, eher von Transparenz zu sprechen, die hinsichtlich der Wirkungsrichtung gewährleistet werden sollte. Wahlsysteme sind komplexe Gebilde, und je mehr Ansprüchen sie gerecht werden sollen, desto weniger leicht verständlich werden sie in ihrem modus operandi. Die personalisierte Verhältniswahl macht da keine Ausnahme. Allerdings muss das Vertrauen bestehen, dass alles mit rechten Dingen zugeht, und dieses Vertrauen wird untergraben, wenn ein

31 Ebenda, Rn. 126.

32 Dolf Sternberger, Über Vorschlag und Wahl. Umriß einer allgemeinen Theorie, in: Karl Heinz Kaufmann / Helmut Kohl / Peter Molt (Hrsg.), Kandidaturen zum Bundestag, Köln 1961, einleitender Essay.

33 BVerfG 2 BvC 1/07 und 2 BvC 7/07, a.a.O. (Fn. 1), Rn. 126; vgl. auch BVerfGE 47, S. 253, S. 279 f.; BVerfGE 95, S. 335, S. 350.

34 BVerfG 2 BvC 1/07 und 2 BvC 7/07, a.a.O. (Fn. 1), Rn. 61. 
Wahlsystem widersinnige Wirkungen zulässt.

\section{Reformalternativen}

Das BVerfG hat in seinem Urteil den Gesetzgeber verpflichtet, eine Wahlsystemreform im Einklang mit der Verfassung herbeizuführen. Um wohl zu verstehen zu geben, was als verfassungskonform in Betracht kommen könnte, hat es Reformmöglichkeiten angedeutet: entweder Verzicht auf Listenverbindungen zur Oberverteilung der Mandate oder Verrechnung der Direktmandate auf Bundesebene, wo die Oberverteilung der Mandate erfolgt, oder Wahl des Bundestages zur einen Hälfte nach Mehrheitswahl und zur anderen Hälfte nach Verhältniswahl (Grabensystem). Mit der Erwägung, auch ein anderer Wahlsystemtyp - und das Grabensystem ist ein solcher - wäre eine verfassungsrechtlich unbedenkliche Option, hat das BVerfG dem Gesetzgeber beachtlichen gestalterischen Spielraum eingeräumt. Da von Seiten des Gerichts nie in Frage gestellt wurde, dass auch ein Mehrheitswahlsystem verfassungskonform sei, scheinen alle Möglichkeiten offen. Stellungnahmen von Persönlichkeiten des öffentlichen Lebens, dies oder jenes Mehrheitswahlsystem einzuführen, nähren ebenfalls diese Annahme.

Die internationale Erfahrung mit Wahlreformen sagt allerdings, dass grundlegende Reformen rar sind. ${ }^{35}$ Dagegen blühen kleine Anpassungen der bestehenden Wahlsysteme an neue Erfahrungen und (leicht) veränderte Zielsetzungen. Solche Reformen haben den Vorteil, dass ihre wahrscheinlichen Effekte besser vorausgesehen werden können - sowohl die gewünschten als auch die berühmt-berüchtigten Nebeneffekte. In Erinnerung sei gerufen, dass alle Wahlsysteme Vorteile und Nachteile haben und dass es kein bestes Wahlsystem gibt, sondern nur ein solches, das am besten passt. Über die geeignete Passform bestimmen allerdings in Reformprozessen weniger die medialen Ideengeber, die gern zu den klassischen Wahlsystemen der Mehrheitswahl greifen, und die Wahlsystemexperten, die das moderne institutional engineering beherrschen, sondern die politischen Parteien. Und die sind hinsichtlich größerer Reformen äußerst zögerlich. Auch bei kleinen Änderungen sind es Machterwägungen, nicht so sehr der mögliche parteiliche Vorteil, sondern die Verhinderung eines Nachteils, die zählen. Eine Reform ist nun jedoch zwingend.

Die naheliegendste Lösung ist sicherlich, die Anrechnung der Direktmandate nicht mehr auf der Ebene der Landeslisten, sondern unmittelbar im Anschluss an die proportionale Zuteilung der Mandate an die Parteien auf nationaler Ebene vorzunehmen. Der Wahlsystemtyp personalisierte Verhältniswahl wäre von einer solchen Reform im Prinzip nicht betroffen. Es wäre die für die Parteien neutralste und die Institutionen schonendste Korrektur des Systems und würde die Vorzüge der personalisierten Verhältniswahl nicht tangieren. Allerdings würde mit der Reform der Verrechnungsweise der Überhangmandate der mandatsrelevante Teil der Verbindung der Personenwahl mit der Verhältniswahl hinfällig, von dem mehrheitsbildende Effekte ausgehen können. Wäre eine solche Minimalreform ausreichend, um aus der Vertrauenskrise des Wahlsystems konstruktiv hervorzugehen?

35 Dieter Nohlen, Changes and Choices in Electoral Systems, in: Arend Lijphart / Bernie Grofman (Hrsg.), Choosing an Electoral System, New York 1984, S. 217 - 224; Arend Lijphart, a.a.O. (Fn. 15); Richard S. Katz, Why are there so Many (or so Few) Electoral Reforms, in: Michael Gallagher / Paul Mitchell (Hrsg.), The Politics of Electoral Reform, Oxford 2008, S. 57 - 79. 


\section{Abschließende Bemerkungen}

Auch wenn die Argumentation des Bundesverfassungsgerichts zu kritisieren ist, geht das erzielte Ergebnis in Ordnung. Das im System der personalisierten Verhältniswahl nicht notwendige Auftreten des negativen Stimmgewichts gehört beseitigt. Sicherlich ist zu würdigen, dass das Gericht erneut die Möglichkeit von Differenzierungen im Wahlsystem anerkannt hat, die Abweichungen vom Erfordernis der Erfolgswertgleichheit darstellen. Es bedarf dafür der Gründe, die von der Verfassung legitimiert sein müssen. „Es genügen in diesem Zusammenhang“, so stellt das Gericht fest, ,auch zureichende, aus der Natur des Sachbereichs der Wahl der Volksvertretung sich ergebende Gründe. Hierzu zählt insbesondere die Verwirklichung der mit der Wahl verfolgten Ziele. Dazu gehören die Sicherung des Charakters der Wahl als eines Integrationsvorgangs bei der politischen Willensbildung des Volkes und die Gewährleistung der Funktionsfähigkeit der zu wählenden Volksvertretung. "36 Es wäre zu wünschen gewesen, dass das Verfassungsgericht diesen allgemeinen, sehr triftigen Überlegungen tatkräftig und konsistent in seiner Argumentation Geltung verschafft hätte. Sie lassen sich mit den von mir aus den internationalen Reformdebatten abstrahierten, als wesentlich betrachteten Wahlsystemfunktionen der Repräsentation (Proporz), Konzentration (Regierbarkeit), Partizipation (Personenwahl), Einfachheit (Transparenz) und Legitimität (verdiente und gezollte Anerkennung) ohne Weiteres verbinden. ${ }^{37}$ Stattdessen orientiert das BVerfG die Bewertung der personalisierten Verhältniswahl an einer einzigen Funktion, der Erfolgswertgleichheit, und radikalisiert sie in etlichen Einzelbegründungen, was zur Begründung des zustande gekommenen Urteils gar nicht nötig gewesen wäre. Dabei verfällt das Gericht zum Teil in eine Argumentationsakrobatik, besonders auffällig in der Begründung für die Verletzung des Grundsatzes der Unmittelbarkeit der Wahl, die ebenso unverständlich wirkt wie der monierte Teil des Wahlsystems nur mit dem Unterschied, dass man ihr rational begegnen kann. Es kann argumentativ nicht um die verstärkte formal-positive Sicherung der Erfolgswertgleichheit gehen, unter anderem wegen der aufgezeigten paradoxen Effekte solcher Maximierungsversuche, sondern nur darum, innerhalb der personalisierten Verhältniswahl rechtspolitisch den inversen Erfolgswert auszuschließen, also zu verhindern, dass die Stimmabgabe für eine Partei eine negative Wirkung zu ihren Lasten entfalten kann, ohne dass der Wähler dies bei seiner Stimmangabe wissen kann.

Wenn der Eindruck nicht täuscht, haben sich Rechtswissenschaftler und Mathematiker immer mehr Fragen des Wahlsystems angenommen. ${ }^{38}$ Wurde dieses Thema in den Anfängen der Bundesrepublik stark von Politikwissenschaftlern einer damals noch jungen Diszi-

36 BVerfG 2 BvC 1/07 und 2 BvC 7/07, a.a.O. (Fn. 1), Rn. 98; vgl. auch BVerfGE 95, S. 408, S. 418.

37 Dieter Nohlen, a.a.O. (Fn. 22), S. 166 - 173.

38 Es ist zu bedauern, dass der anerkannte juristische Wahlsystemspezialist Hans Meyer nicht als Sachverständiger, sondern als Bevollmächtigter, also als Anwalt der Beschwerdeführer aufgetreten ist. Als einziger Sachverständiger war ein Mathematiker geladen, was nicht gegen den Kollegen Friedrich Pukelsheim spricht, der ausgezeichnete Arbeit im Bereich der mathematischen Aspekte der Wahlsystemforschung leistet, die inzwischen mit dem Max-Planck-Forschungspreis ausgezeichnet wurde. 
plin sowohl in normativen als auch in empirischen Bezügen behandelt, ${ }^{39}$ die allesamt Mehrheitswahl oder mehrheitsbildende Elemente im Wahlsystem favorisierten, so hat sich das Bild inzwischen völlig verschoben. Die Zurückhaltung der Politikwissenschaftler (von Eckard Jesse und anderen abgesehen ${ }^{40}$ ) hat sicherlich damit zu tun, dass sich die personalisierte Verhältniswahl bewährt hat und inzwischen international zu einem Modellwahlsystem aufgestiegen ist, in einigen Ländern sogar übernommen und in Neuseeland der traditionellen relativen Mehrheitswahl vorgezogen wurde. ${ }^{41}$ In Großbritannien sprach sich die bislang letzte Wahlreformkommission 1998 für ein dem deutschen System nachempfundenes kombiniertes Wahlsystem aus. ${ }^{42}$ Während in der Politikwissenschaft die Frage des Wahlsystems also zur Ruhe kam - Dolf Sternberger meinte Ende der 1970er Jahre, dass man aufgrund der Einübung der Spielregel keine grundstürzende Veränderung mehr vornehmen sollte -, blieben Rechtswissenschaftler und Mathematiker am Ball, erstere als Folge der Wahleinsprüche gegen „Ungereimtheiten“ der personalisierten Verhältniswahl, die sich aus der Verbindung der verschiedenen Elemente von Entscheidungsregel (nach der Mehrheit in den Einerwahlkreisen) und Repräsentationsprinzip (auf der Ebene der Zusammensetzung des Parlaments nach Parteien) ergaben. Das Bundesverfassungsgericht war mit seinen Urteilen zur Sperrklausel, zum Zweistimmensystem, zu den Überhangmandaten maßgeblich an diesem Vorrang beteiligt. Die Mathematiker wiederum entdeckten die Verrechnungsverfahren bei Proporzwahlen als empirische „Spielwiese“ ihrer mathematischen Modelle. Während international die große Mehrheit der Länder, in denen nach Verhältniswahl gewählt wird, entweder das System d'Hondt oder das einfache Wahlzahlverfahren (Hare plus größter Überrest) anwenden, mithin einfach nachvollziehbare Verrechnungsverfahren, wurde in Deutschland das Verfahren Hare/Niemeyer eingeführt, von dem es jetzt heißt, es führe zu paradoxen Ergebnissen, sodann im Jahre 2008 das Verfahren Sainte-Laguë/Schepers, das sehr kompliziert ist, mit Auf- und Abrundungen arbeitet und das Zahlenmaterial so lange schüttelt, bis der angeblich beste Proporz dabei herauskommt. ${ }^{43}$ Rechtswissenschaftler und Mathematiker haben mit der Erfolgswertgleichheit eine gemeinsame idée fixe.

39 Vgl. Ferdinand A. Hermens, Demokratie oder Anarchie. Untersuchungen über die Verhältniswahl, Köln / Opladen 1968; Dolf Sternberger, Die große Wahlreform, Opladen 1964; Rudolf Wildenmann / Werner Kaltefleiter / Uwe Schleth (Hrsg.), Auswirkungen von Wahlsystemen auf das Parteien- und Regierungssystem der Bundesrepublik, in: Erwin Scheuch / Rudolf Wildenmann (Hrsg.), Zur Soziologie der Wahl, Köln / Opladen 1965, S. 74 - 112.

40 Eckard Jesse, Reformvorschläge zur Änderung des Wahlrechts, in: APuZ, B 52 (2003), S. 3 - 11.

41 Vgl. Peter Lösche, Do Electoral Systems Matter? Überlegungen am Beispiel Neuseelands, in: ZParl, 35. Jg. (2004), H. 2, S. $340-358$.

42 Vgl. Dieter Nohlen, a.a.O. (Fn. 22), S. 303 f.

43 Vgl. Friedrich Pukelsheim, Mandatszuteilungen bei Verhältniswahlen: Vertretungsgewichte der Mandate, in: KritV, 83. Jg. (2000), H. 1, S. 76 - 103. Das Zusammenspiel ergibt sich, indem der Mathematiker vom Bundesverfassungsgericht den ,zentralen Maßstab“ der Erfolgswertgleichheit der Stimmen übernimmt und folgert: „Gleichheit als abstraktes Postulat verpflichtet, die real unvermeidlichen Ungleichheiten so klein wie möglich zu halten“ (S. 79). Dann werden die Methoden auf diese Eigenschaft hin miteinander verglichen und diejenige vorgeschlagen, die der vorgegebenen „Verpflichtung“ am besten nachkommt. Sie findet bei Juristen und juristisch geschulten Beamten Anklang. Ob jemand die 2008 eingeführte „Divisormethode mit geometrischer Rundung" noch verstehen und in der politischen Bildungsarbeit erklären kann und sie mehr Vertrauen bei den Bürgern in den Wahlakt weckt, wird kaum reflektiert. 
Wenn man berücksichtigt, dass angesichts der Entwicklung eines Fünfparteiensystems in Deutschland weniger Stimmenanteile auf jene beiden Parteien entfallen, die bisher fast ausschließlich in der Lage waren, Direktmandate zu erringen, so wird erkennbar, welche mehrheitsbildende Komponente in Form von Direktmandaten das bundesdeutsche Wahlsystem gerade in einer Zeit verliert, wo diese zur Mehrheitsbildung gut würden beitragen können. Denn es ist jenseits aller Erklärungsfeinheiten in der Entstehung von Überhangmandaten ${ }^{44}$ jedermann einsichtig, dass bei starker Konzentration der Stimmen auf zwei (oder zweieinhalb) Parteien wenige Überhangmandate entstehen, sie aber in dem Maße zunehmen, wie der Gesamtstimmenanteil der beiden großen Parteien sinkt, ohne dass dritte Parteien Direktmandate erzielen können. Deshalb ist es angebracht, bei der Reform des Wahlsystems nicht nur eine „verfassungsgemäße Regelung“ herbeizuführen, sondern auch an eine Substituierung des jetzt entfallenden Mehrheitselements zu denken, das gerade dann eine relative mehrheitsbildende Wirkung zu entfalten in der Lage wäre, wenn der Proporz (trotz Geltung einer Sperrklausel, die heutzutage in fast allen Verhältniswahlsystemen gang und gäbe ist) der Dekonzentration im Parteiensystem und den erhöhten Schwierigkeiten parlamentarischer Mehrheitsbildung freien Lauf lässt. Für den Verlust der Überhangmandate als mandatsrelevanter Ressource der „vorgeschalteten Mehrheitswahl“ verdient die personalisierte Verhältniswahl eine die parlamentarische Mehrheitsbildung fördernde Kompensation, um Regierbarkeitsprobleme wie in der Weimarer Republik nicht aufkommen zu lassen.

44 Vgl. Florian Grotz, Die personalisierte Verhältniswahl unter den Bedingungen des gesamtdeutschen Parteiensystems. Eine Analyse der Entstehungsursachen von Überhangmandaten seit der Wiedervereinigung, in: PVS, 41. Jg. (2000), H. 4, S. 707 - 729; Joachim Behnke, Von Überhangmandaten und Gesetzeslücken, in: APuZ, B 52 (2000), S. 21 - 28. 